\title{
PID Parameters Optimization and Its Engineering Application
}

\author{
Li Yongling ${ }^{1, a}$, Yang Zuoliang ${ }^{1, b}$ \\ ${ }^{1}$ State Grid Jibei Electric Power Co. Ltd. Skill Training Center,Baoding, Hebei , 071051, China; \\ lyl8012131@163.com
}

Keywords: PID parameters optimization, PSO, Engineering application

\begin{abstract}
The performance of PID controller depends on the combination of the control parameters. This paper puts emphasis on the study of particle swarm optimization (PSO) in parameter optimization for PID controller. Firstly, according to a great amount of experiment, a set of formulas were proposed in dealing with PID controller optimization for self-balanced and non-self-balanced objects respectively. Then corresponding optimization procedures had been programmed with PSO, and tests show the procedures are effective.
\end{abstract}

\section{Introduction}

PID controller, with its simple structure, stability, reliable and easy to adjust, becomes one of the main techniques of industrial control. PID controller is based on deviation, using proportional, integral and differential of a system to calculate the control parameters. The performance of PID controller depends on the combination of the control parameters. The existing methods used to tune PID controller include Ziegler-Nichol (Z-N), critical proportional band, simplex search, and so on, but the regulation effect is not well ${ }^{[1]}$.

Particle swarm optimization (PSO) is a multi-agent parallel search technique. Kennedy and Eberhart introduced the concept of function-optimization by means of a particle swarm [2,3]. This paper puts emphasis on the study of PSO in parameter optimization for PID controller. Firstly, according to a great amount of experiment, a set of formulas were proposed in dealing with PID controller optimization for self-balanced and non-self-balanced objects respectively. Then corresponding optimization procedures had been programmed with PSO.

\section{PID Optimization Algorithm}

\subsection{Characteristics of thermal objects}

There are two types of objects in terms of the characteristics of response to the input variations [4]. Firstly, after the disturbances of energy and substances, if the objects reach a new stability level, they will be called self-balanced objects. Secondly, if the objects cannot recover from the disturbances or develop with a constant speed at a new stability level, they will be non-self-balanced objects.

Thermal self-balanced objects can generally be described as the following transfer function.

$$
G=\frac{K}{(T s+1)^{n}} e^{-\tau s}
$$

For non-self-balanced objects, the function is:

$$
G=\frac{K}{s(T s+1)^{n}} e^{-\tau s}
$$

In these formulas, there are four parameters to be explained, and they are respectively: $K$ -Steady-state gain;T - Time constant;n - Order; $\tau$ - Delay time.

\subsection{PID parameters tuning}

It has been found that there is a certain relationship between the parameters of the controller and those of the objects to be controlled. There have been formulas about the controller parameters' setting such as Ziegler-Nichol (Z-N) method, and these formulas based on the test of step response. This method has been widely used in engineering field and process control. 
Based on many years of experiences and simulation experiment, we have concluded another set of formulas concerning the parameters of the objects. And the formulas based on practical experiences are showed below.

$$
\left\{\begin{array}{l}
K_{P}=1 /[0.3 * K *(n+1) \\
T_{i}=20+0.5 *(n-1) * T+0.5 * \tau \\
T_{d}=0.25 * T
\end{array}\right.
$$

In these formulas, every parameter belonging to the object is taken into consideration to decide the satisfactory of the controller parameters, while in $\mathrm{Z}-\mathrm{N}$ method the order $\mathrm{n}$ is not correlative in determining the controller parameters. And it is clear that this set of formulas are simple to calculate and being programmed.

\subsection{Optimization of PID parameters based on PSO}

PSO is a new evolutionary algorithm developed recently. As genetic algorithms(GA), PSO starts from a random solution, and finds the optimal solution by iteration. It evaluates the quality of the solution through a fitness, therefore it is much simpler than genetic algorithm. There isn't "cross" and "mutation" operation, finding the global optimum by following the optimal value of the current search[5].

Assuming there is a group with m particles in D dimensional target search space, the position of the i-th particle can be represented as a vector:

$$
x_{i}=\left(x_{i 1}, x_{i 2}, \ldots, x_{i D}\right), i=1,2, \cdots, m
$$

It's speed is also a $D$ dimensional vector:

$$
v_{i}=\left(v_{i 1}, v_{i 2}, \cdots, v_{i D}\right)
$$

The best position of $i$-th particle is:

$$
P_{g}=\left(p_{g 1}, p_{g 2}, \cdots, p_{g D}\right)
$$

PSO formula is as follows:

$$
\begin{gathered}
v_{i d}(t+1)=v_{i d}(t)+c_{1} r_{1}\left(p_{i d}-x_{i d}(t)\right)+c_{2} r_{2}\left(p_{g d}-x_{i d}(t)\right) \\
x_{i d}(t+1)=x_{i d}(t)+v_{i d}(t+1)
\end{gathered}
$$

Where, $\mathrm{i}=1,2, \ldots, \mathrm{m}, \mathrm{D}$. The acceleration constant $C_{1}$ and $C_{2}$ is none negative constant; $r_{1}$ and $r_{2}$ are random numbers and they are distributed in $[0,1]$. vid(t) is current position of the $\mathrm{i}$-th particle, Pid is the current velocity of the $\mathrm{i}$-th Particle.

When $v_{i d}>V_{\text {max }}$, take $v_{i d}=V_{\text {max }}$; When $v_{i d}<-V_{\text {max }}$, take $v_{i d}=-V_{\text {max }}$. Vid $\$$ max,Vmax], Vmax is Maximum speed limit, and it's non-negative.

\section{Engineering Implementation}

For control system, when the set value changed or the disturbance of the system occurred, the system will go to a new steady state from the original steady state by a process. The quality of an automatic control system is difficult to determine in the steady state, and it could only be adequately reflected in the transition process. When perturbation occurred, a good control system should be smooth, rapid and accurate approaching or back to the settings, and this requires accurate settings for the control parameters of the PID controler to achieve the desired results. The PID controllers at the factory are generally not provide ready-made parameters, so we use PSO to optimize the PID control system, and a corresponding optimization program was built.

\subsection{Single-loop System}

Single-loop control system is the simplest, most basic and most widely used form. There are two forms of control object:N-order inertia plus pure delay; The one-order inertia series.

$\mathrm{N}$-order inertia with pure delay transfer function is chosen as controlled object:

$$
G=\frac{1.267}{(18.4 s+1)^{6}} e^{-10 s}
$$


The result of simulation is shown in Figure1, and Tab.1 is the performance parameters of control system which have shown in interface.

Table 1: The program output list of single loop system

\begin{tabular}{c|c|c|c|c|c}
\hline$K_{\mathrm{P}}$ & $K_{\mathrm{I}}$ & $\mathrm{D}$ & Overshoot & Adjustment time (s) & Decay rate \\
\hline 0.49527 & 0.00676 & 0 & $8.83 \%$ & 452.64 & $90.18 \%$ \\
\hline
\end{tabular}

While the transfer function of controlled object is changed into:

$$
G=\frac{1.202}{(27.1 s+1)^{7}}
$$

The result of simulation is shown in Figure2, and Tab.2 is the performance parameters of system as formula (10).

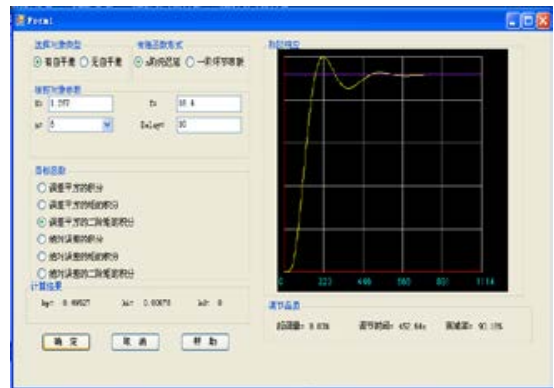

Fig.1 Result of procedure

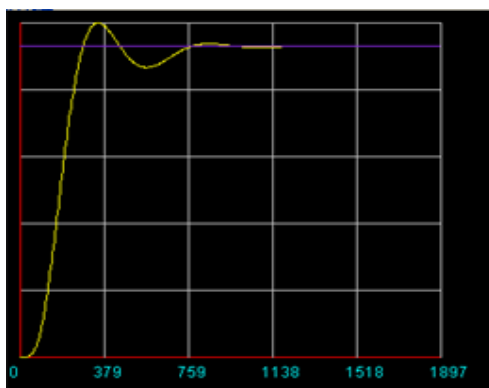

Fig.2 Result of procedure

Table 2: The program output list of single loop system

\begin{tabular}{c|c|c|c|c|c}
\hline \hline$K_{\mathrm{P}}$ & $K_{\mathrm{I}}$ & $K_{\mathrm{D}}$ & Overshoot & Adjustment time (s) & Decay rate \\
\hline 0.52168 & 0.00444 & 0 & $7.34 \%$ & 717.07 & $90.92 \%$ \\
\hline \hline
\end{tabular}

\subsection{Cascade control system}

The cascade control system includes two control loops: the main loop and vice loop, which is shown in Figure 3. The vice loop composed of measured variable transmitter, vice regulator, adjustable valve and vice processes, and the main loop composed of main measured variable transmitter, main regulator, adjustable valve and main processes. The forms of control object are also divided into n-order inertia with pure delay and one-order inertia series.

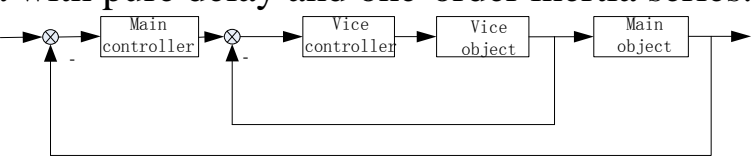

Fig. 3 Cascade control system diagram

\subsubsection{N-order inertia with pure delay}

The tested objects are:

$$
\begin{aligned}
G_{\text {main }} & =\frac{-1.657}{(20 s+1)^{2}} \\
G_{\text {vice }} & =\frac{-1.657}{(20 s+1)^{2}}
\end{aligned}
$$

The simulation result of procedure is shown in Fig. 4, and the performance parameters of system are shown in Tab.3.

\subsubsection{The one-order inertia series}

The form of control object not only may be the expression of n-order inertia with pure delay, but also the combination of two forms of object. Transfer function of vice loop:

$$
G_{\text {vice }}=\frac{1.202}{(18 s+1)(18.4 s+1)(20 s+1)(27.1 s+1)}
$$

Transfer function of main loop: 


$$
G_{\text {main }}=\frac{1.202}{(27.1 s+1)^{7}} e^{-5 s}
$$

The simulation result of procedure is shown in Fig.5, and the performance parameters of system are shown in Tab. 4

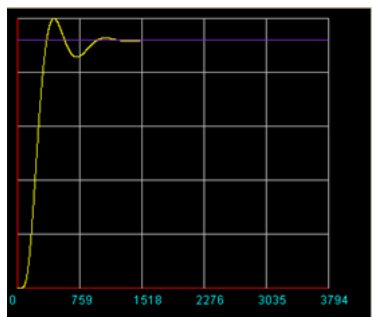

Fig.4 Double loop system step response curve order

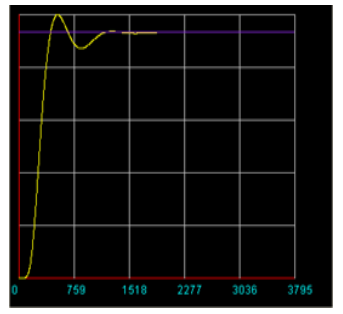

Fig. 5 Double loop system step response curve order

Table 3: PID parameters of cascade system

\begin{tabular}{c|c|c|c}
\hline \hline PID parameters & $K_{\mathrm{P}}$ & $K_{\mathrm{I}}$ & $K_{\mathrm{D}}$ \\
\hline Main loop & 0.5128 & 0.0035 & 0 \\
\hline Auxiliary loop & -0.6706 & -0.0134 & 0 \\
\hline \hline \multicolumn{2}{|c|}{ Table 4: PID parameters of cascade system } & $K_{\mathrm{I}}$ \\
\hline \hline PID controller parameters & $K_{\mathrm{P}}$ & 0.0028 & 0.5096 \\
\hline Main loop & 0.5251 & 0.0078 & 0 \\
\hline Auxiliary loop & 0.5546 & & $K_{\mathrm{D}}$ \\
\hline \hline
\end{tabular}

\section{Conclusion}

Although the PID control system has experienced so many years of development and has been very mature. Because of its wide range of industrial automation applications, the optimization problem of PID controller parameters has been attract more and more attention. In order to solve this problem, this paper put forward PSO to optimize the PID parameters and programmed corresponding procedures. Tests show that the procedures are effective References.

\section{References}

[1] Liu L., Mao Z. Water turbines PID controller based on genetic algorithm[J]. Automation of Electric Power Systems, 1997, 21 (12):41-43.

[2] M.Dorigo. V.Maniezzo and A.Colorni. Evolutionary Algorithms in Engineering and Computer Science, John Wily \& Sons Ltd, England, 1999.

[3 ]Dorigo M, Caro G D. Ant colony optimization: a new meta-heuristic. Proceeding of the 1999 Congress on Evolutionary Computation, Washington DC: USA 1999, 1470-1477.

[4] Park J B, Lee K S, Shin J R, et al. A particle swarm optimization for economic dispatch with nonsmooth cost functions [J]. IEEE Trans on Power Systems, 2005,20(1):34-42.

[5] Qian Zhang, Ze Dong, Pu Han, Zhongli Wu, Fang Gao. Optimization of Controllers in the Thermal System Using Initial Pheromone Distribution in Ant Colony Optimization. IEEE International Conference on Information Reuse and Integration, Las Vegas, 2008. 